\title{
Investigation of the Magnetosome Biomineralization in Magnetotactic Bacteria Using GLC-TEM
}

Emre Firlar ${ }^{1,2}$, Meagan Ouy ${ }^{1}$, Agata Bogdanowicz ${ }^{1}$, Leigha Covnot ${ }^{1}$, Boao Song ${ }^{2}$, Yash Nadkarni ${ }^{1}$, Reza Shahbazian-Yassar ${ }^{2}$ and Tolou Shokuhfar ${ }^{1}$

1. University of Illinois at Chicago, Department of Bioengineering, Chicago, IL.

2. University of Illinois at Chicago, Department of Mechanical and Industrial Engineering, Chicago, IL.

Understanding the biomineralization pathway for the magnetosomes in magnetotactic bacteria is of utmost importance for the biomimicking of the $\mathrm{Fe}_{3} \mathrm{O}_{4}$ magnetosome synthesis ex situ due to their potential uses in physical and medical sciences. Until now, the approaches involved conventional and fluid flow holder approaches, which have disadvantages of not keeping the bacterial cells viable and having reduced imaging spatial resolution due to excess liquid thickness, respectively ${ }^{1-3}$. To overcome this, we encapsulated magnetotactic bacteria in graphene liquid cells (GLC) right after mixing them with iron rich growth medium as shown in Figure $1 \mathrm{~A}^{4-6}$. Here, by maintaining the native environment of the AMB-1 bacterium, we were able to monitor for the first time with GLC-TEM imaging the formation of these nanoparticles and increased nanoparticle contrast due to advancing biomineralization (Figure 1B). Encapsulation of bacterial culture in graphene and preservation of growth medium surrounding the bacterium with intact graphene were verified via low loss electron energy loss spectroscopy (EELS). Characterization of the mature magnetosomes was carried out via $\mathrm{L}_{3}$ core edge EELS.

Verification of the intact graphene and water in the growth medium was proved by carrying out low loss EELS. Low loss EELS data shows the presence of graphene optical gap at $6 \mathrm{eV}$, water exciton peak at $8.5 \mathrm{eV}$ and graphene $\sigma+\pi$ bond at $14 \mathrm{eV}$. Further investigation of the chemical environment in the magnetosomes was carried out by iron $\mathrm{L}_{3}$ core edge EELS peak fitting analysis as shown Figure $1 \mathrm{C}^{1,7}$. Formation of radiation induced $\mathrm{H}_{2}$ bubbles and magnetosomes are shown with white and black arrows, respectively. The collected spectrum was fitted with reference spectra of $\mathrm{Fe}^{2+}$ (octahedral), $\mathrm{Fe}^{3+}$ (tetrahedral), $\mathrm{Fe}^{3+}$ (octahedral) and $\mathrm{FeOOH}$. Relative ratio of $\mathrm{Fe}^{2+}$ to $\mathrm{Fe}^{3+}$ help investigate the final structure of the magnetosomes when kept properly in the growth medium. The ratio for the mature magnetosome here is reported as 0.35 . Considering that ratio will be 0.5 for a perfect $\mathrm{Fe}_{3} \mathrm{O}_{4}\left(1 \mathrm{x} \mathrm{Fe}{ }^{2+}, 2 \mathrm{x}\right.$ $\left.\mathrm{Fe}^{3+}\right), 0.35$ suggests the presence of another type iron oxide which contributes to higher percentage of $\mathrm{Fe}^{3+}$. Hematite is the strongest candidate for this iron oxide. As suggested earlier by Firlar et al. through Gibbs Free Energy calculations, magnetite might have oxidized to maghemite and with the electron beam exposure, the maghemite might have converted to hematite ${ }^{4}$.

The increase of the magnetosome image contrast was reported by the line profile drawn across the magnetosomes. Comparison of the image contrast via line profiles drawn across the magnetosomes at $\mathrm{t}: 17$ minutes after induction with $\mathrm{t}: 31$ minutes after induction showed increased mass-thickness related TEM contrast in the image, which indicates that this particular magnetosome progressed more in its biomineralization path as time passes, which is, the formation of more $\mathrm{Fe}_{3} \mathrm{O}_{4}$ in this particle. Formation of new magnetosomes were also reported throughout GLC-TEM images on a particular bacterium. This shows the applicability of GLC-TEM technique for nano-scale monitoring sub-cellular activities while keeping the cells viable [8]. 
References:

[1] E Firlar et al, J. Mater. Res. 31 (2016), p. 547.

[2] TJ Woehl et al, Microsc. Microanal. 22 (2016), p. 12.

[3] TJ Woehl et al, Sci. Rep. 4 (2014), p. 6854.

[4] E Firlar et al, Microsc. Microanal. 23 (2017), p. 1310.

[5] C Wang et al, Adv. Mater. 26 (2014), p. 3410.

[6] C Wang T Shokuhfar and RF Klie, Adv. Mater. 28 (2016), p. 7716.

[7] E Firlar et al, Sci. Rep. 5 (2015), p. 9830.

[8] The authors acknowledge the NSF CAREER Award DMR-1564950, NSF-DMR Award number 1710049 and Chicago Biomedical Consortium Award PDR-086 for financial support.
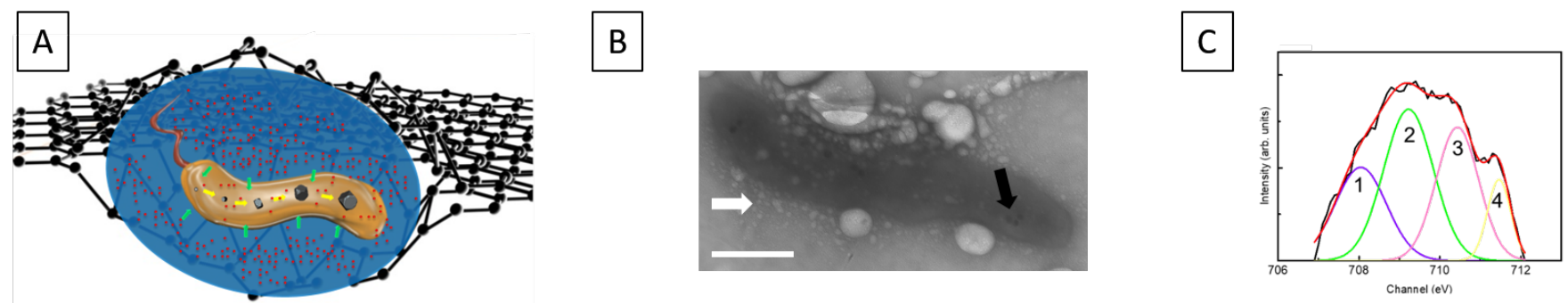

Figure 1. A) Schematic showing in situ bacterial magnetosome biomineralization phenomenon in GLC setup. Red dots are $\mathrm{Fe}^{2+}$ ions. Green arrows indicate internalization of $\mathrm{Fe}^{2+}$ ions. Yellow arrow shows the biomineralization pathway B) GLC-TEM image of AMB-1 bacterium, Scale bar: $500 \mathrm{~nm} \mathrm{C)} \mathrm{Fe} \mathrm{L}_{3}$ core EEL spectrum of a fully grown bacterium is reported. The ratio of the areas of peak 1 to peaks $(2+$ 3 ), corresponding to $\mathrm{Fe}^{2+}$ (octahedral) to $\mathrm{Fe}^{3+}$ (tetrahedral + octahedral) ratio, is around 0.35 . Peak 4 is attributed to the $\mathrm{FeO}(\mathrm{OH})$. 